Ger J Exerc Sport Res 2021 · 51:202-212 https://doi.org/10.1007/s12662-021-00709-7 Eingegangen: 19. Oktober 2020

Angenommen: 12. Januar 2021

Online publiziert: 16. Februar 2021

(c) Der/die Autor(en) 2021

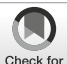

Die Entwicklung des Bobsports von einer unterhaltsamen Freizeitaktivität für wohlhabende Touristen in St. Moritz Ende des 19. Jahrhunderts bis zum Hochleistungssport der Gegenwart ist neben vielem anderen durch eine Regeländerung aus dem Jahr 1952 gekennzeichnet, die für diese Sportart bis heute zentral ist. „In 1952, a critical rule change limiting the total weight of crew and sled ended the era of the super heavyweight bobsledder and sealed the future of the sport as an athletic contest of the highest caliber" (IBSF, 2020a). Die Festlegung des Maximalgewichts von Schlitten und Team ${ }^{1}$ hatte zur Folge, dass die ganz „schweren Jungs “ aus dem Bobsport verschwanden, stattdessen zunehmend starke und schnelle Athleten aus anderen Sportarten rekrutiert wurden und vor allem die Startphase erheblich an Bedeutung gewann. Die Aktiven des Bobsports sind sich seit Langem darin einig, wie der ehemalige Schweizer Viererbobfahrer Thomas Handschin sagt, dass der „Bobstart [... ein wesentlicher Faktor für eine gute Endzeit [ist]“ (Handschin, o.J.). Noch deutlicher formuliert es der Bob- und Schlittenver-

\footnotetext{
1 Gemäß dem Bob-Reglement der "International Bobsleigh \& Skeleton Federation“ (IBSF) beträgt das Maximalgewicht im Zweierbob Männer $390 \mathrm{~kg}$ (170 kg der Schlitten), im Viererbob Männer $630 \mathrm{~kg}$ (210 Kg der Schlitten), im Zweierbob Frauen $330 \mathrm{~kg}$ (170 kg der Schlitten) (IBSF, 2020b, S. 31) sowie im Monobob Frauen - einer erstmals in der Saison 2020/2021 in den Weltcup aufgenommenen Disziplin - $247 \mathrm{~kg}$ (162 kg der Schlitten) (IBSF, 2020c, S.6).

2 "Schwere Jungs" (2006) ist der Titel eines Spielfilms von Marcus Rosenmüller über das deutsche Viererbobteam bei den Olympischen Winterspielen 1952 in Oslo, das dort Gold gewann, weil es auf den Positionen zwei bis vier die schwersten,Athleten' sitzen hatte.
}

\author{
Annika Elm ${ }^{1} \cdot$ Robert Gugutzer ${ }^{2}$ (D) \\ ' Deutscher Olympischer Sportbund (DOSB), Frankfurt, Deutschland \\ ${ }^{2}$ Institut für Sportwissenschaften, Goethe-Universität Frankfurt a.M., Frankfurt a.M., Deutschland
}

\title{
Der Viererbobstart
}

\section{Eine leibphänomenologische Analyse}

band für Deutschland (BSD) auf seiner Homepage: „Ein schneller Start ist ausschlaggebend, da die Entscheidung über die Erstplatzierung in den meisten Fällen in der Anschub- und Startphase fällt" (BSD, o.J.).

Vor dem Hintergrund der offenkundig zentralen Bedeutung der Startphase im Bobsport ist es bemerkenswert, wie wenig sich die Sportwissenschaft mit ihr befasst hat. Wissenschaftliche Studien zum Bobsport sind zum einen im Bereich der Sporttechnologie und Sportgeräteforschung verortet, wo beispielsweise Fragen zur Aerodynamik des Schlittens oder der Kufen-Eis-Reibung im Mittelpunkt stehen (Boerboom, Hainzlmaier, Kraus, \& Wintermantel, 2006; Dabnichki, 2015; Hainzlmeier, Wolf, \& Wintermantel, 2005; Pernpeintner \& Winkler, 2010; Poirier, Lozowski, Maw, Stafnyshyn, \& Thompson, 2011; Winkler \& Pernpeintner, 2008). Im deutschen Bobsport spielt diesbezüglich das „Institut für Forschung und Entwicklung von Sportgeräten“ (FES) eine maßgebliche Rolle. Zum anderen werden trainingswissenschaftliche, biomechanische und sportmedizinische Fragestellungen etwa zur reaktiven Bewegungsleistung (Kümmel et al., 2015), zum Kraft-, Schnelligkeits- und Techniktraining von Bobfahrern (Gerber, 1987) oder zu Verletzungen und gesundheitlichen Risiken im Bobsport (Freimüller, Biener, \& König, 2000) verfolgt. Die wenigen sportwissenschaftlichen Untersuchungen, die explizit die Startphase im Bobsport zum Gegenstand haben, sind ebenfalls primär trainingswissenschaftlicher und biomechanischer Art und thematisieren zum Beispiel die für die Beschleunigung relevanten Faktoren
Kraft, Schnelligkeit, Kondition und Koordination (Dabnichki, 2016; Goldmann et al., 2018; Leopold, 2020; Meyer, 2008; Park, Lim, \& Kim, 2019), den Einfluss der Eis-Metall-Reibung beim Anschieben des Schlittens (Cross et al, 2017) oder die unter aerodynamischem Gesichtspunkt optimale Körperhaltung und Sitzposition im (Zweier-)Bob (Winkler \& Pernpaintner, 2010).

Die disziplinären Zugänge und thematischen Schwerpunkte der wissenschaftlichen Analysen des Bobsports bringen es mit sich, dass eine naheliegende Frage anscheinend gar nicht erst gestellt wird: Wie gelingt der Bobstart? Auch wenn die Startphase bei dem in diesem Text ausschließlich interessierenden Viererbob lediglich fünf bis sechs Sekunden dauert, sollte es unstrittig sein, dass hier vier Athleten ${ }^{3}$ sowohl ihr Verhalten untereinander als auch im Zusammenspiel mit einem schweren Sportgerät auf eisglattem Untergrund $k o$ ordinieren müssen. Dass diese MenschMensch- und Mensch-Material-Koordination gelingt, ist nun aber keineswegs selbstverständlich. Sportwissenschaftliche Abhandlungen hierzu liegen gleichwohl nicht vor. Womöglich hat dies damit zu tun, dass die Einschätzung des Biomechanikers Peter Dabnichki keine bloße Einzelmeinung, sondern Mehrheitsmeinung ist: „Bobsleigh start is a simple action requiring the crew to push as hard as possible and gain maximum initial velocity of the sled at the start" (Dabnichki, 2016, S. 193).

\footnotetext{
3 Da der Viererbob - warum auch immer den Männern vorbehalten ist, verwenden wir im Weiteren ausschließlich die männliche Schreibweise "Athlet ${ }_{\text {, }}$,Sportler" etc.
} 
In dem vorliegenden Beitrag verzichten wir auf die Bewertung, ob der Viererbobstart einfach oder schwierig ist. Wir setzen stattdessen einen Schritt davor an, indem wir herausarbeiten, wie das Zusammenspiel von MenschMensch und Mensch-Material beim Viererbobstart erfolgt. Es geht uns mithin um das „doing bobsleigh start", um das interaktive Geschehen der Athleten miteinander sowie zwischen ihnen und ihrem Sportgerät. Unser Erkenntnisziel ist dabei ein hermeneutisches und kein sportpraktisches: Wir wollen verstehen, wie der Viererbobstart vollzogen wird, aber wir wollen keine Handlungsempfehlungen geben, wie er vollzogen werden könnte oder gar sollte.

Den Weg zu diesem Erkenntnisziel ebnet uns die Phänomenologie, konkret die Leibphänomenologie des Kieler Philosophen Hermann Schmitz. Wir wählen eine leibphänomenologische Analyse des Viererbobstarts deshalb, weil bei diesem - so unsere These - der Wahrnehmung und dem subjektiven Erleben der Athleten eine herausragende Bedeutung zukommt. Wir gehen davon aus, dass es aufgrund der sehr kurzen Dauer der Startphase für die Athleten nahezu unmöglich ist, ihr Handeln zu reflektieren und sich sprachlich zu verständigen. Für ihr gemeinschaftliches Tun ist stattdessen ein „Koagieren ohne Reaktionszeit" (Schmitz, 1985, S. 86) kennzeichnend, und dies nicht nur zwischenmenschlich, sondern auch transhuman, nämlich mit dem Schlitten (und teilweise auch mit dem Eis). Entscheidend für den Viererbobstart ist, mit Schmitz gesprochen, die „leibliche Kommunikation" (Schmitz, 1990, S. 135-153) zwischen den Athleten einerseits und den Athleten mit ihrer dinglich-materiellen Umwelt andererseits. In diesem Sinne geht es hier nicht allein um das "doing“, sondern ebenso sehr um das „feeling bobsleigh start“. Unsere zentrale These lässt sich daher auch so formulieren: Für das Gelingen eines Viererbobstarts sind nicht allein die Körper der Athleten (Kraft, Schnelligkeit etc.) und die sportartspezifischen Dinge (Schlitten, Schuhe, Eis etc.) ausschlaggebend, sondern gleichermaßen die Leiblichkeit der Athleten.
Im Folgenden stellen wir zuerst die für unsere Arbeit zentralen Begriffe der Leibphänomenologie von Schmitz vor, daran anschließend die empirische Grundlage unserer Untersuchung - qualitative Interviews mit acht Athleten des BSD sowie unser methodisches Vorgehen. Im Mittelpunkt steht sodann die leibphänomenologische Analyse und Interpretation des Viererbobstarts. Im Schlusskapitel fassen wir die zentralen Ergebnisse der Studie zusammen und betonen die Erkenntnisrelevanz des Beitrags für eine empirische Sportphilosophie, insbesondere für die phänomenologische Sportforschung.

\section{Grundzüge der Leibphäno- menologie von Hermann Schmitz}

Es gibt eine Vielzahl an leibphänomenologischen Ansätzen (vgl. Alloa, Bedorf, Grüny, \& Klass, 2012; Rappe, 2018), unter denen die Leibphänomenologie von Hermann Schmitz aufgrund ihrer begrifflichen Differenziertheit und thematischen Vielfalt heraussticht. Dass sie im Vergleich zu den Leibphänomenologien von beispielsweise Max Scheler, Erwin Straus, Jean-Paul Sartre und ganz besonders Maurice Merleau-Ponty einen deutlich geringeren Bekanntheitsgrad hat, dürfte primär ${ }^{4}$ damit zu tun haben, dass Schmitz in vielerlei Hinsicht mit der Tradition der Phänomenologie bricht ${ }^{5}$ bezeichnenderweise nennt er seinen Ansatz „Neue Phänomenologie“ (Schmitz, 2003, 2009). Mit Blick auf den Leib zeigt sich der Traditionsbruch etwa daran, dass Schmitz nicht - wie Husserl - von der „Intentionalität“ des Leibes und dem „fungierenden Leib“ oder - wie MerleauPonty - dem leiblichen „Zur-Welt-Sein“

\footnotetext{
${ }^{4}$ Die geringe Bekanntheit von Schmitz in der internationalen Philosophie und anderen Disziplinen hat darüber hinaus sicherlich auch damit zu tun, dass von ihm erst eine englischsprachige Buchpublikation vorliegt, und die zudem erst seit Kurzem (Schmitz, 2019). 5 Auf die Unterschiede zwischen Schmitz' Leibphänomenologie und anderen Leibphänomenologien kann hier aus Platzgründen nicht eingegangen werden. Siehe dazu neben der bereits genannten Literatur auch Böhme (2003, Teil 1) oder Fuchs (2000, Kapitel 2).
}

spricht, sondern vom „affektiven Betroffensein“ oder leiblichen "Ergriffensein“. Schmitz' Leibbegriff rückt damit - wie ansatzweise auch jener von Straus, Sartre und Bernhard Waldenfels - die pathische Dimension des Leiblichen in den Mittelpunkt, konkret: das Spüren. Schmitz definiert:

Leiblich ist, was jemand in der Gegend (keineswegs, wie z.B. am Blick deutlich wird, immer in den Grenzen) seines materiellen Körpers von sich selbst [...] spüren kann, ohne sich der fünf Sinne (Sehen, Tasten, Hören, Riechen, Schmecken) und des aus ihrem Zeugnis abgeleiteten perzeptiven Körperschemas (der habituellen Vorstellung vom eigenen Körper) zu bedienen. Als Leib kann dann das Ganze der leiblichen Regungen mit seiner [...] räumlichen und dynamischen Beschaffenheit verstanden werden [...]. (Schmitz, 2011, S. 5; Herv. im Orig.).

Das Zitat verdeutlicht zum einen, dass Leib, Sinne und Körper - zu ergänzen wären noch die Gefühle ${ }^{6}$ - begrifflich auseinanderzuhaltende Phänomene sind. Leiblich ist all das, was man an sich spürt, und das ist weder identisch mit dem, was man sieht, tastet, hört, riecht oder schmeckt, noch mit dem materiellen Körper, der als „sinnfälliger“ Körper an seiner Oberfläche sicht- und tastbar und im naturwissenschaftlichen Verständnis mess- und quantifizierbar ist (Schmitz, 2011, S. 143). Der Körper kann zudem wie ein Instrument oder Werkzeug (aktiv) eingesetzt werden, wohingegen der Leib sich als Widerfahrnis (passiv) bemerkbar macht. Zum anderen ist in dem Zitat angedeutet, dass Schmitz' Leibbegriff nicht auf eine Wesensbestimmung abzielt, sondern auf eine strukturelle Kennzeichnung. Nach Schmitz ist der Leib eine räumlich-dynamische Struktur, deren basales Kategorienpaar der spürbare Gegensatz von „Enge“ und „Weite“ ist (ebd., S. 7-27). Das Einatmen

\footnotetext{
6 Schmitz definiert "Gefühl" als "räumlich ausgedehnte Atmosphäre" und unterscheidet zwischen Gefühl und dem Fühlen des Gefühls, wobei er für Letzteres zwei Varianten kennt: das affektive Betroffensein vom Gefühl und das bloße Wahrnehmen des Gefühls (Schmitz, 2003, S.44).
} 
oder ein Muskelkrampf sind spürbar als Engeempfinden, das Ausatmen (bis zu einem gewissen Punkt) und die Auflösung des Krampfes als Weiteempfinden. Ein-/ Ausatmen sowie Krampf/Entkrampfung sind räumliche Phänomene, da sie ein spürbares (flächenloses) Volumen haben, und sie stehen in einem dynamischen, einander abwechselnden Verhältnis mal überwiegt die Enge, mal die Weite. Schmitz zufolge ist dieser innerleibliche Dialog von „Engung“ (bzw. „Spannung“) und „Weitung“ (bzw. „Schwellung“) der „vitale Antrieb“ des Menschen (ebd., S. 15) - ein grundsätzliches Merkmal menschlichen Daseins im Wachzustand.

Wesentlich für Schmitz' Leibphänomenologie ist nun, dass der Leib nicht etwas rein Individuelles oder bloß Innerliches ist, sondern ein das Individuum überschreitendes Phänomen. Schmitz spricht von der „Exteriorisierung des innerleiblichen Dialogs“ (Schmitz, 1985, S. 85) und meint damit, dass der innerleibliche Dialog von Engung und Weitung in einen transleiblichen Dialog übergehen kann, wodurch ad hoc ein übergreifender Leib entsteht. Schmitz nennt dieses Phänomen, dass sich der vitale Antrieb eines leiblichen Wesens (Mensch, Tier) mit einem anderen leiblichen Wesen oder etwas Leiblosem (Dingen, Räumen, Atmosphären etc.) verbindet, „leibliche Kommunikation“ bzw. „Einleibung“ (Schmitz, 2011, S. 29-53). Hierbei unterscheidet er zwei Hauptformen: Die Einleibung ist ,antagonistisch“, wenn einer der Kommunikationspartner leiblich dominiert (wobei die leibliche Dominanz dauerhaft oder fluktuierend sein kann), die leiblichkommunikative Beziehung also durch Überlegenheit und Unterworfensein gekennzeichnet ist (Ringkampf, Blickkontakt); „solidarisch“ ist die Einleibung, wenn die Beziehung der Kommunikationspartner eine Art Miteinander ohne leibliche Dominanz auf der einen oder anderen Seite ist, sodass mehrere Leiber durch einen gemeinsamen vitalen Antrieb zu einer leiblichen Einheit zusammengeschlossen werden (Rudern im Achter, La-Ola im Stadion). Die antagonistische Einleibung kann wiederum „einseitig“ oder „wechselseitig“ sein. Sie ist einseitig, wenn ausschließlich

Ger J Exerc Sport Res 2021 · 51:202-212 https://doi.org/10.1007/s12662-021-00709-7 (c) Der/die Autor(en) 2021

\section{A. Elm · R. Gugutzer}

\section{Der Viererbobstart. Eine leibphänomenologische Analyse}

\section{Zusammenfassung}

Im Bobsport herrscht Konsens, dass die Startphase von zentraler Bedeutung für eine gute Endzeit ist. Dennoch hat sich die Sportwissenschaft mit der Frage, wie der Bobstart gelingt, bis dato kaum auseinandergesetzt. Der Beitrag holt dies in Form einer leibphänomenologischen Analyse der Startphase im Viererbob nach, indem er sein Augenmerk sowohl auf die leibliche Kommunikation der Athleten untereinander als auch zwischen ihnen und ihrem Sportgerät richtet. Theoretisches Fundament hierfür ist die Leibphänomenologie von Hermann Schmitz, empirische Grundlage sind problemzentrierte Interviews mit acht Kaderathleten des Bob- und Schlittenverbands für Deutschland (BSD). Zentrales Ergebnis der Untersuchung ist erstens, dass für das Gelingen des Viererbobstarts vor allem die antagonistisch-einseitige Einleibung der
Athleten untereinander wie auch der Athleten mit dem Bob bedeutsam ist. Zweitens erweist sich die solidarische Einleibung der Athleten als wichtige Bedingung und gleichermaßen spürbarer Ausdruck eines gelungenen Viererbobstarts. Der Text präsentiert damit einen vollkommen neuen Blick auf den Bobsport. Mit seinem theoretisch-konzeptionellen Fokus auf leibliche Kommunikation im Sport liefert er darüber hinausgehend einen innovativen Beitrag zur phänomenologischen Sportforschung, wie er generell die fruchtbare Verbindung von Philosophie und empirischer Sportwissenschaft belegt.

\section{Schlüsselwörter}

Bobsport · Leibphänomenologie · Leib · Leibliche Kommunikation · Hermann Schmitz . Phänomenologische Sportforschung

\section{The four-man bobsleigh start. An analysis from the perspective of felt-bodily phenomenology}

\section{Abstract}

In bobsleigh sport, there is a consensus that the start phase is crucial for a good finish time. Yet, sport science has hardly dealt with the question of how the bobsleigh start succeeds. This article contributes to this topic in the form of a neo-phenomenological analysis of the start phase in four-man bobsleigh by focusing on the felt-bodily communication of the athletes with each other as well as between them and their sports equipment. The theoretical foundation for this is the phenomenology of felt-body by Hermann Schmitz, the empirical basis are problem-centered interviews with eight squad athletes of the German Bobsleigh and Luge Association (Bob- und Schlittenverband für Deutschland). The central result of the study is: First, the one-sided antagonistic encorporation between the athletes as well as between the athletes and the bobsled is significant for the success of the fourman bobsleigh start. Second, the solidary encorporation of the athletes proves to be an important condition as well as a tangible expression of a successful four-man bobsleigh start. Thus, the article presents a completely new view of the sport of bobsleigh. With its theoretical-conceptual focus on felt-bodily communication in sports, it also makes an important contribution to phenomenological sport research, as it generally demonstrates the beneficial link between philosophy and empirical sport science.

\section{Keywords}

Bobsleigh · New Phenomenology · FeltBody - Felt-bodily communication - Hermann Schmitz · Phenomenological sport research einer der Beteiligten die leibliche Dominanzrolle innehat, so dass der andere gewissermaßen an ihm hängt (Faszination durch spannendes Spiel, Suggestion des Balls oder Faustschlags, der auf einen zukommt); wechselseitig ist die Einleibung, wenn die leibliche Dominanzrolle zwischen den Kommunikationspartnern hin und her pendelt (Führen und Folgen im Paartanz, Ballwechsel beim Tennis). Die antagonistisch-einseitige Einleibung ist so gesehen monologisch, die antagonistisch-wechselseitige Einleibung dialogisch.

Ein wichtiges Merkmal leiblicher Kommunikation ist darüber hinaus, 
dass sie vermittelt vonstattengeht, und zwar insbesondere dann, wenn kein Körperkontakt zwischen den Kommunikationspartnern herrscht. Schmitz nennt solche Kommunikationsmedien „leibnahe Brückenqualitäten“ (ebd., S. 33). Er meint damit „Bewegungssuggestionen“, das heißt Vorzeichnungen von Bewegungen wie den Rhythmus oder einen Gestaltverlauf(Flanke im Fußball), sowie „synästhetische Charaktere“, womit Wahrnehmungsqualitäten gemeint sind, die die verschiedenen Sinnesgebiete verbinden (leicht/schwer, hell/dunkel, warm/kalt, weich/hart etc.).

In unserer Analyse des Viererbobstarts nutzen wir vorrangig das Konzept der leiblichen Kommunikation, da es mit ihm möglich ist, nichtsprachliche Verständigungs- und präreflexive Abstimmungsprozesse, wie sie für den Viererbobstart charakteristisch sind, phänomengerecht zu beschreiben. Wir betreten damit relatives Neuland in der Sportwissenschaft, da hier weder Schmitz' pathischer Leibbegriff noch sein Konzept der leiblichen Kommunikation sonderlich zur Kenntnis genommen werden, geschweige denn, dass mit ihnen gründlich gearbeitet wird. $^{7}$

\section{Methodisches Vorgehen}

Empirische Datengrundlage des vorliegenden Beitrags sind qualitative Interviews mit acht deutschen Bob-Athleten ${ }^{8}$. Der Feldzugang erfolgte über eine E-Mail-Anfrage bei einem Funktionär des BSD, der den Kontakt zu einem Trainer und dieser wiederum den Kontakt

\footnotetext{
7 Selbstredend gibt es Ausnahmen, zum Beispiel Ute Ewalds Studie zum Hallenklettern (Ewald, 2015), Florian Hartnacks Untersuchung zum Kampfsport in der Schule (Hartnack, 2017) oder Michael Staacks Studie zu Mixed Martial Arts (Staack, 2019). Siehe zudem die neophänomenologischen Arbeiten des Autors dieses Beitrags beispielsweise zur Sportsucht (Gugutzer 2015a), zur leiblichen Interaktion mit Sportgeräten und -elementen (Gugutzer 2015b), zu den Atmosphären des Sports (Gugutzer 2020), etwa beim Public Viewing (Gugutzer 2015c), zum Fitness-Tracking (Gugutzer 2016) oder zu den Moden des Sports (Gugutzer 2019).

${ }^{8}$ Die Interviews entstammen der Masterarbeit der Autorin dieses Beitrags (Elm 2020).
}

zu den Athleten herstellte. Die Athleten gehören dem Olympia-, Perspektiv- und Ergänzungskader des BSD an und haben nationale sowie internationale Erfolge auf allen Wettkampfebenen erzielt. Die Interviews fanden in der Vorbereitungsphase der Saison 2019/2020 im Rahmen eines Lehrgangs an einer deutschen Eisbahn statt, und zwar im unmittelbaren Anschluss an die Trainingsläufe der Athleten. Die Interviews wurden mit einem digitalen Aufnahmegerät aufgezeichnet, in Standardorthografie transkribiert und vollständig anonymisiert.

Die Datenerhebung wie auch die Datenanalyse erfolgten in Anlehnung an das problemzentrierte Interview (Witzel \& Reiter, 2012), die Dateninterpretation theoriegeleitet auf der Grundlage der Leibphänomenologie von Schmitz (s. oben). Für die Datenerhebung bot sich das problemzentrierte Interview an, da der Gegenstand unserer Untersuchung ein soziales Problem ist (Wie gelingt der Viererbobstart?), es einen Leitfaden enthält, der für die Forschungsfrage relevante Themenbereiche bündelt, sowie gleichermaßen erzählungsgenerierende Fragen und (immanente und exmanente) Nachfragen integriert. Auf diese Weise konnte sichergestellt werden, dass die Interviewpartner möglichst präzise erzählen, was sie beim Bobstart tun und wahrnehmen. In der Datenanalyse stand zunächst die fallspezifische („vertical“) und anschließend die fallübergreifende („horizontal“) „identification of key themes“ (Witzel \& Reiter, 2012, S. 107) im Mittelpunkt. Das Ergebnis der Datenanalyse unterscheidet sich dabei von den im Leitfaden festgelegten Themenbereichen: War der Leitfaden thematisch in die drei großen Blöcke „MenschMensch-Interaktion“, „Mensch-Maschine-Interaktion“ und „Mensch-UmweltInteraktion" unterteilt, so ergab sich aus der Datenanalyse die thematische Struktur „Einstimmen“, „Anschieben“, „Einsteigen“, „Hinsetzen“. Die Datenanalyse trägt damit der Relevanzsetzung der Interviewpartner Rechnung, für die die phasenspezifische Unterteilung des Bobstarts bedeutsamer ist als eine Differenzierung nach Interaktionspartnern.

Die theoriegeleitete Dateninterpretation implizierte schließlich zweierlei. Ers- tens fungierte die Leibphänomenologie als eine Art ,Lesebrille‘, die es ermöglicht, ,hinter' den Worten der Interviewpartner den Leib zu ,sehen'. Das bedeutet, wir interpretieren Äußerungen wie „da merk ich, das was nicht stimmt" oder „der Schlitten geht dann zu schwer“ und metaphorische Ausdrücke wie „der Tank ist leer" oder „bin so wie ein Stier, der losgelassen wird" als sprachliche Hinweise auf leibliche Phänomene. Aus Sicht eines Athleten - Leiblichkeit gibt es nur in der Ersten-Person-Perspektive (vgl. Bedorf, 2017, S. 73) - bedeutet „merken“, dass er etwas leiblich an sich wahrnimmt, wie sich auch der „schwergängige“ Schlitten, der „leere Tank“ und der „losgelassene Stier" dem Athleten als spürbare Last, Schwäche und leiblicher Antrieb zeigen. ${ }^{9}$ Diente die theoriegeleitete Dateninterpretation somit erstens dazu, induktiv aus dem Datenmaterial heraus relevante leiblich-kommunikative Aspekte des Viererbobstarts offenzulegen, so war ihr zweiter Zweck deduktiver, konkret subsumtionslogischer Art: Die im ersten Interpretationsschrittherausgearbeiteten leiblich-kommunikativen Phänomene subsumierten wir in zwei Kategorien, die wir mit Schmitz als antagonistische und solidarische Einleibung bezeichnen. In Kombination mit den vier zeitlichen Teilphasen ergeben diese zwei leiblich-kommunikativen Dimensionen die zentralen leibphänomenologischen Merkmale des Viererbobstarts.

\section{Leibliche Kommunikation beim Viererbobstart}

Im Folgenden stellen wir die leibphänomenologischen Merkmale des Viererbobstarts vor (siehe zusammenfassend

\footnotetext{
9 Im traditionellen phänomenologischen Verständnis ist ein Phänomen, wie Heidegger sagt, "das, was sich zeigt, das Sichzeigende, das Offenbare" (Heidegger 2006, S. 28). Das, was sich zeigt, kann auch etwas sein, das man spürt. Im neophänomenologischen Verständnis hingegen ist ein Phänomen „für jemand zu einer Zeit [..] ein Sachverhalt, dem der Betreffende dann nicht im Ernst den Glauben verweigern kann, dass es sich um eine Tatsache handelt" (Schmitz, 2009, S. 12). Schmitz' Phänomenbegriff unterscheidet sich von jenem Heideggers somit durch eine doppelte, nämlich personale und zeitliche, Relativierung.
} 


\section{Leibliche Kommunikation Startphasen}

\begin{tabular}{|c|c|c|c|c|c|}
\hline & & Einstimmen & Anschieben & Hineinspringen & Hinsetzen \\
\hline \multirow[t]{2}{*}{$\begin{array}{l}\text { Antago- } \\
\text { nistische } \\
\text { Einlei- } \\
\text { bung }\end{array}$} & einseitig & $\begin{array}{l}\text { Durch Musik „pushen“, in } \\
\text { „Flow“ geraten, „,hochfah- } \\
\text { ren”, sich „fokussieren“ }\end{array}$ & $\begin{array}{l}\text { Sehen des Piloten, Hören } \\
\text { der Ausrufe; Spüren des „zu } \\
\text { leichten/schweren“ Schlit- } \\
\text { tens; zeitliche Unstimmigkeit } \\
\text { merken }\end{array}$ & $\begin{array}{l}\text { Bewegungsaufforderungen } \\
\text { von Abweiser und Bord- } \\
\text { wand; Bewegungsimpulse } \\
\text { „übertragen“, Kette von } \\
\text { bilateralen Bewegungssug- } \\
\text { gestionen der Fahrer }\end{array}$ & $\begin{array}{l}\text { Hintermann (dessen Füße) } \\
\text { "erahnen”, „spüren“; visuelle, } \\
\text { akustische und spürbare } \\
\text { Bewegungssuggestionen } \\
\text { der Fahrer }\end{array}$ \\
\hline & wechselseitig & $\begin{array}{l}\text { Gegenseitig „Power" an } \\
\text { Teammitglieder weiterge- } \\
\text { ben, leibliche Ansteckung }\end{array}$ & $\begin{array}{l}\text { Leibliche Abstimmung (Kor- } \\
\text { rektur) bei wahrgenomme- } \\
\text { nem „Hinterherhängen“ }\end{array}$ & & \\
\hline \multicolumn{2}{|c|}{ Solidarische Einleibung } & $\begin{array}{l}\text { Empfundene Solidargemein- } \\
\text { schaft durch Rhythmus des } \\
\text { Abklatschens }\end{array}$ & $\begin{array}{l}\text { "gut eingespieltes" Team, } \\
\text { "gemeinsamer Rhythmus"; } \\
\text { "Gesamtimpuls" durch pas- } \\
\text { sendes „Timing" bzw. gleich- } \\
\text { zeitiges „Treffen" am Bügel }\end{array}$ & $\begin{array}{l}\text { "Timing-Sache“, „gut einge- } \\
\text { spieltes“ Team }\end{array}$ & $\begin{array}{l}\text { Gleichzeitiges Hinsetzen } \\
\text { (,Timing") von Position 2, } 3 \\
\text { und } 4\end{array}$ \\
\hline
\end{tabular}

- Tab. 1). Aus Platzgründen fokussieren wir hierbei ausschließlich den Viererbobstart bei Wettkämpfen und nicht im Training. Leibphänomenologisch ist das bedeutsam, da sich der Viererbobstart in einem Wettkampf von jenem im Training in leiblicher Hinsicht beispielsweise dadurch unterscheidet, dass die Athleten anders gekleidet sind (spürbar weite statt spürbar enganliegende Kleidung) und die Anspannung geringer ist.

\section{Einstimmen}

Im Unterschied zum Training beginnt die Startphase bei einem Wettkampf bereits kurz vor dem eigentlichen, durch ein akustisches (Ton) und visuelles (Ampel) Signal angezeigten Start. Die Athleten stimmen sich hier sowohl individuell als auch gemeinsam auf den Start ein. Eine typische Praxis der individuellen Einstimmung besteht darin, sich einen Kopfhörer aufzusetzen und (laute) $\mathrm{Mu}$ sik zu hören. Zweck des Musikhörens ist es, „fokussierter" (IP_AD, Z. 157) ${ }^{10}$ zu sein, sich zu „pushen“ (ebd., Z. 150) oder in einen „Flow“ (IP_AA, Z. 202) zu geraten. Dazu ein Zitat von IP_AD:

Eigentlich habe ich immer schon meine Kopfhörer drin. Die sind auch dann auf Anschlag aufgedreht, weil ich sage mal, ich eher das Probleme habe mich zu pushen. Also das vermeintliche Durchdrehen, nen-

10 Wir zitieren die Interviewpartner anonymisiert mit ihrem Codenamen und den Zeilennummern des Interviewtranscripts. ne ich es jetzt mal. Dass man halt wirklich Gas geben kann. Ich habe da schon so ein bisschen rumprobiert, auch mit ruhiger Musik, aber das ist dann eher in die Hose gegangen. Dann fahre ich noch mehr runter. Ja und dann habe ich eher das Problem, das persönliche Problem, dass ich so aus der Haut fahre. Das, was natürlich beim Sprint oder hier natürlich von Vorteil wäre, genau deswegen. Da bin ich jetzt draufgekommen, habe dann ein Lied auf Dauerschleife, was dann läuft auf Anschlag. Und ich sage mal, umso näher dass dann der Start kommt, umso fokussierter werde ich dann (IP_AD, Z. 149-157).

Der Bobstart im eigentlichen Sinne beginnt mit einem Sprint. Um diesen mit maximaler Schnellkraft starten zu können, ist es notwendig, dass die Athleten sich in einem spezifischen leiblichen $\mathrm{Zu}$ stand befinden, den man mit Schmitz als maximale „leibliche Intensität“ bezeichnen kann (Schmitz, 1965, S. 120). Leibliche Intensität ist das spürbare Resultat des simultanen Zusammenspiels von Engung (Spannung) und Weitung (Schwellung) und ein konstitutives Element jeder Kraftanstrengung. Wer sich in einem dösigen, meditativen Zustand befindet, wird nicht in der Lage sein, zusammen mit anderen ein $210 \mathrm{~kg}$ schweres Sportgerät schnell fortzubewegen. In dem Zitat ist nun zu erfahren, dass dieser Athlet das Problem hat, sich „pushen“ $\mathrm{zu}$ müssen. Andere Bobathleten mögen dieses Problem eines reduzierten vitalen Antriebs zwar nicht haben, aber auch sie pushen sich typischerweise vor dem Start und streben „so dieses Hochfahren“ (IP_AA, Z. 198) an. „Pushen“ und „Hochfahren“ sind aus Sicht des Betroffenen leiblich engende Regungen, das von diesem Athleten ebenfalls erwähnte „Runterfahren“ ist eine leiblich weitende, „protopathische“ (Schmitz, 2011, S. 24) Regung. Gemeinsam ist dem leiblichen Hoch- und Runterfahren, dass sie, wie das Zitat belegt, manipulierbar sind. In antagonistisch-einseitiger leiblicher Kommunikation mit der Musik - im Schmitz'schen Sinne einem „Halbding“ (Schmitz, 2003, S. 14) - beeinflusst der Bobfahrer seinen leiblichen Zustand. „Auf Anschlag aufgedrehte", also laute (und vielleicht auch schnelle und/oder harte) Musik affiziert ihn auf eine stimulierende Weise, sodass er körperlich „Gas geben“ kann; „ruhige“ (und vielleicht auch leise und/ oder sanfte) Musik greift hingegen in seinen leiblichen Haushalt auf eine seinen vitalen Antrieb dämpfende Weise ein. Die antagonistisch-einseitige Einleibung mit lauter Musik hat zudem - sicherlich nicht nur - bei ihm den positiven leiblichen Effekt einer stärkeren „Fokussierung “ auf den Start. Wer fokussiert ist, befindet sich in einer für die Bewegungsausführung förderlichen, weil engenden leiblichen Konzentration.

Neben der antagonistisch-einseitigen leiblichen Einstimmung ist für Bobteams kurz vor dem Start ebenso eine antagonistisch-wechselseitige Form stimmungsvoller Einleibung charakteristisch. Bobteams haben typische Einstimmungsrituale, unter denen das 
gegenseitige „Abklatschen“ von besonderer Bedeutung ist. Dazu exemplarisch IP_BB:

Also erst mal probieren wir relativ [...] unser Abklatschkommando durchzuziehen, dass das dann weg ist. Das motiviert uns gegenseitig auch noch mal ganz gut, dass wir da so ein bisschen uns - ja, wir klatschen uns ja ab in einem gewissen Rhythmus und das gibt mir eigentlich immer viel Power auch. Ich hoffe, ich gebe meine Power auch so ein bisschen nach außen. Dass die Jungs merken - okay, der ist willig, der ist bereit. Das ist die Intention, für die man das eben macht dann quasi. (IP_BB, Z. 37-42)

Das Abklatschen ist eine ritualisierte Praxis, die sich in ihrer körperlichen Ausführung zwar von Bobteam zu Bobteam leicht unterscheidet (Reihenfolge der Akteure, nur gegenseitiges High Five, auf Helm und/oder Rücken schlagen etc.), hinsichtlich der damit verbundenen „Intention“ aber identisch ist. Dem Zitat zufolge ist mit dem Abklatschen eine doppelte, nämlich psychische und leibliche Intention verbunden: Das wechselseitige Abklatschen soll „motivieren“ und „Power" generieren. Entscheidend ist hierfür der „Rhythmus“, in dem die Athleten ihre körperliche Interaktion vollziehen: Der Rhythmus fungiert als „leibnahe Bewegungssuggestion zur Anstiftung solidarischer Einleibung " (Schmitz, 2011, S. 48). Im Medium des rhythmischen Abklatschens entsteht im Bobteam ad hoc ein übergreifender Gesamtleib, ein sich wechselseitig stärkendes, leibliches Kollektiv. Diese solidarische Einleibung basiert auf einer antagonistisch-wechselseitigen Einleibung, nämlich der Weitergabe der subjektiv erlebten Power "nach außen“ an die Teammitglieder, die diese Power „merken“, also spüren (sollen). Der körperlich hergestellte, transleibliche Rhythmus zeigt sich für die einzelnen Akteure als spürbare Kraft/Stärke/Macht, die sie im Sinne einer gegenseitigen leiblichen Ansteckung aneinander weitergeben mit dem doppelten Effekt, eine empfundene Solidargemeinschaft zu sein, in der jeder Einzelne bestmöglich motiviert ist.

\section{Anschieben}

Unmittelbar auf das Einstimmen folgt die Phase des Anschiebens, die damit beginnt, dass die vier Athleten ihre jeweilige Startposition einnehmen: Der Pilot (Position 1) geht an den vordersten Anschubbügel, die beiden Anschieber (Position 2 und 3) an den linken und rechten Anschubbügel und der Bremser (Position 4) am Schlittenende an die zwei Anschubgriffe; jeder Fahrer umfasst mit beiden Händen seinen Bügel bzw. seine Griffe. Die beiden Anschieber und der Bremser stehen dabei auf dem eisfreien Startbalken, der Pilot befindet sich bereits auf der Eisbahn. Anschließend erfolgt ein teaminternes Startkommando, das aus drei bis vier Wörtern besteht, die abwechselnd vom Bremser und Piloten gerufen werden. Zum Beispiel:

$\mathrm{Na}$, der Bremser sagt „steht", wenn der sieht, dass ich [= Position 1] vorne stehe und so leicht nach vorne gucke. Das ist so ganz gut eingespielt. Dann wissen die hinten - aha, es geht los, der ist bereit. Dann sagt der halt "steht", dann sage ich "fertig“" und dann gibt es so ein bisschen ein leicht längeres „und“ und auf das „d“ sollen eigentlich alle die maximale Power auf die Bügel bringen. (IP_BD, Z. 120-124)

Das Startkommando beginnt im Fall dieses Bobteams mittels einer antagonistisch-einseitigen Einleibung, wenn man mit Schmitz davon ausgeht, dass die „normale Wahrnehmung [...] in erster Linie Einleibung“ ist, und daher auch „Sehen Einleibung ist“ (Schmitz, 1990, S. 138). Der Bremser „sieht“, dass der Pilot vorne steht und nach vorne guckt und reagiert darauf mit dem Ausruf „steht“. Dieser Ausruf wird vom Piloten (und den beiden Anschiebern) gehört und unmittelbar mit dem Ausruf „fertig“ beantwortet, was wiederum vom Bremser gehört und mit einem langezogen „und“ beantwortet wird. Beim Hören der Wörter „steht“, „fertig“, „und“ handelt es sich ebenfalls um eine Einleibung, da es hier nicht so sehr auf das Verstehen der inhaltlichen Bedeutung der Wörter ankommt (das Kommando könnte sicherlich genauso gut „eins“, „zwei“, „drei“ lauten), sondern auf den Rhythmus, in dem sie geäußert werden. Die rhythmische Schal- läußerung fungiert hierbei als Medium solidarischer Einleibung. Diese setzt umgekehrt aber auch voraus, wie es in dem Zitat heißt, dass das Team "gut eingespielt" ist. Ist dies der Fall, entspricht das Startkommando einer habitualisierten Form leiblicher Kommunikation, deren soziale Funktion darin besteht, dass „alle die maximale Power auf die Bügel bringen“. Dies ist die zentrale Aufgabe beim Anschieben: Alle vier Athleten sollen mit maximaler Kraft den Schlitten beschleunigen - und zwar im selben Moment.

Also das Timing vom Treffen ist natürlich auch immens wichtig. Wenn alle vier gleich viel Kraft auf einmal aufwenden, geht der Schlitten natürlich viel besser weg, wie wenn es nacheinander ist oder die Seitenleute zuerst. Deswegen ist dieser Gesamtimpuls, der da kommt, richtig wichtig. (IP_AC, Z. 273-276)

Der Übergang vom Startkommando zum Anschieben erfolgt durch eine zeitliche Abstimmung („Timing“) der vier Athleten, die im Idealfall $\mathrm{zu}$ einem gleichzeitigen „Treffen“ an den Anschubbügeln und -griffen und infolgedessen einem gleichzeitigen Loslaufen führt. Diese zeitliche Abstimmung basiert auf einer solidarischen Einleibung des Teams, einem „Koagieren ohne Reaktionszeit" (Schmitz, 1990, S. 137), das nur gut eingespielte Teams hinbekommen. „Wer oben am Balken großartig nachdenkt“, so IP_AA (Z. 243), wird $\mathrm{zu}$ früh oder zu spät loslaufen und das Anschieben damit negativ beeinflussen. $\mathrm{Ob}$ das Timing gepasst hat, ist für die vier Akteure spürbar. Sie merken beim Anschieben nicht nur in antagonistischeinseitiger Einleibung, dass der Schlitten ein schwergewichtiger Interaktionspartner ist, dem mit „roher Gewalt“ (IP_BC, Z. 129) oder dadurch zu begegnen ist, dass man „wie so ein Stier, der der dann losgelassen wird“ (IP_AC, Z. 193), also mit maximaler leiblicher Intensität eine maximale körperliche Kraftanstrengung ausübt. Sondern ebenso spürbar ist für die Athleten das Leichte oder Schwere des angeschobenen Schlittens und damit das Ge- oder Misslingen ihres gemeinsamen Anschiebens. 
Also man kriegt schon mit, [...] ob alle vier sich getroffen haben. Oder ob einer ein bisschen hinterherhängt oder ob man selber ein bisschen zu schnell war. Das kriegt man mit, indem es entweder zu leicht geht. Wenn es zu leicht geht, ist es meistens so, dass man selber ein bisschen hinterherhängt. Beim Kommando, dass man zu spät an den Schlitten heranläuft. Oder halt logischerweise umgekehrt, wenn man zu zeitig ran läuft, dass man auf einmal die ganze Last auf einem hat. Und dann merkt man, wenn die anderen mitschieben, dann wird's halt schlagartig leichter. (IP_BC, Z. 198-205)

Dass das Anschieben nicht gelungen ist, zeigt sich für die Athleten mittels einer leiblichen Differenzerfahrung: ${ }^{11}$ Fühlt sich das Anschieben zu leicht an, ist man zu spät dran, fühlt es sich zu schwer an, ist man zu früh dran. Die synästhetischen Charaktere "leicht" und "schwer" fungieren hierbei als Medien ein- und wechselseitiger leiblicher Kommunikation. In antagonistisch-einseitiger Einleibung spürt der jeweilige Fahrer das Leichte oder Schwere des Schlittens am eigenen Leib; in antagonistisch-wechselseitiger Einleibung spürt der Einzelne entweder, „wenn die anderen mitschieben“, weil es "dann schlagartig leichter" wird (nachdem er selbst zu früh dran war), oder aber er spürt, dass er "hinterherhängt" (er also zu spät dran war), was bei ihm den leiblichen Impuls auslöst, „schnell [zu] reagieren“, damit „man dann in dem ganzen Rhythmus mit reinkommt“ (IP_BC, Z. 215f.). Die leibliche Dominanzrolle haben in diesem Fall zunächst die Teammitglieder inne, bei denen das Timing gepasst hat, insofern sie den zu spät losgelaufenen Kameraden unwillentlich leiblich dazu drängen, dass er sein Fehlverhalten korrigiert. Dessen

11 Leibliche Differenzerfahrungen sind in der Startphase auch in der Auseinandersetzung mit der Umwelt möglich, etwa aufgrund synästhetischer Charaktere der Lufttemperatur und/oder der Eisoberfläche: „Wenn die Luft trocken ist und es ist so knapp unter null Grad, dann hat man eigentlich die besten Bedingungen. Weil wenn es noch kälter ist, dann klebt der Schlitten meist am Start, dann ist der Start ein bisschen langsamer." (IP_BD, Z. 49-51; Herv. von Verf.). „schnelles Reagieren“ bedeutet sodann einen kurzfristigen Wechsel der leiblichen Dominanzrolle auf seine Seite, wo sie jedoch nicht bleibt, da es darum geht, in einen gemeinsamen "Rhythmus" zu kommen. Das heißt: Obgleich die Zeitspanne des Anschiebens extrem kurz ist, ist es den Athleten möglich, eine zeitliche Unstimmigkeit im gemeinsamen Bewegungsablauf leiblich (irritiert) wahrzunehmen und unmittelbar körperlich handelnd auszubessern. Sofern dies gelingt, ist die antagonistisch-wechselseitige in eine solidarische Einleibung übergegangen.

\section{Hineinspringen}

Die Phase des Anschiebens geht fließend über in jene, in der die vier Athleten in der Reihenfolge ihrer Positionen in den fahrenden Bob hineinspringen: Die "Eins“ (Pilot) beginnt, ihm folgen die "Zwei“, die „Drei“ und zuletzt die „Vier“ (Bremser). Die Athleten bezeichnen diese Phase als die schwierigste des Starts, weil sie „nicht komplett automatisiert" (IP_AB, Z. 294) abläuft. Die größten Herausforderungen haben dabei die Anschieber auf den Positionen 2 und 3 zu meistern. Es stellen sich für sie vor allem zwei potenzielle Probleme, die aus der antagonistisch-einseitigen Einleibung von Athlet und Schlitten resultieren:

Einerseits kann man bei diesem Brett unten abrutschen tatsächlich, wenn man es nicht richtig trifft oder wenn der Schuh voller Eis ist eventuell. Das nächste ist, man könnte an der Bordwand hängenbleiben, wenn man ein bisschen nachlässig ist oder eben die Füße hängen oder so was. (IP_BB, Z. 208-212)

Die „Seiteneinsteiger“, wie sie sich selbst nennen, haben als Erstes die Aufgabe, mit dem schlittenfernen Fuß auf den Abweiser („Brett unten“) zu springen. Aus der Ersten-Person-Perspektive des Seiteneinsteigers ist der Abweiser ein leiblich machtvoller Partner, da er ihn nötigt, etwas Bestimmtes zu tun, eben mit dem äußeren Fuß auf das Brett zu springen. Konkret handelt es sich hier um eine „sozialisierte“ bzw. „habitualisierte Nötigung" (Gugutzer, 2015b, S. 111), haben die Athleten doch durch ihr wiederhol- tes Training das leibliche Wissen erworben, den Appell des Abweisers mit genau diesem Verhalten zu erwidern. Im Wettkampf wird diese körperliche Erwiderung mitunter durch den leiblichen Umstand erschwert, dass am Ende der Anschiebephase der vitale Antrieb der Athleten nahezu erschlafft ist. Es ist schwierig, so ein Seiteneinsteiger, wenn „ich alles, was ich im Tank habe, auf den Metern bis dahin rausgeknallt habe, $[\ldots]$ trotzdem nochmal voll konzentriert sein muss und in den Schlitten reinkommen muss"(IP_AB, Z. 288-290). Weil am Ende der Beschleunigungsphase der "Tank“ leer ist, was subjektiv vielleicht als, schwere Beine' oder Kurzatmigkeit spürbar ist, ist die Konzentration auf das richtige Treffen des Abweisers, das Nichtabrutschen und das Hineinspringen in den Bob eine Herausforderung.

Wie das Zitat andeutet, ist außerdem das Überwinden der Bordwand keine Selbstverständlichkeit, vielmehr kann es passieren, dass man mit den Füßen an der Bordwand „hängenbleibt“. So erweist sich auch die Bordwand als eine leiblich machtvolle Antagonistin, da sie den Athleten nötigt, seine Beine auf eine Höhe zu heben, die es ihm ermöglicht, störungsfrei in den Bob zu springen. Für den gelingenden Seiteneinstieg ist es damit erforderlich, dass die Athleten die Bewegungsaufforderungen des Abweisers und der Bordwand, die sie leiblich wahrnehmen und von denen sie leiblich gefordert werden (Konzentration), mit einem adäquaten körperlichen Verhalten $\mathrm{zu}$ erwidern. ${ }^{12}$

\footnotetext{
12 Die antagonistisch-einseitige Einleibung zwischen Athlet und Schlitten ist mitunter auch durch die Lateralität des Athleten beeinflusst. Davon erzählt IP_AB, der sich als Rechtshänder eindeutig "auf der Drei links" wohler fühlt als rechts vom Schlitten: „[...] wenn ich von der linken Seite schiebe, ne, dann habe ich das Gefühl, ich habe eine Stabilität, weil der Bügel hier innen ja fixiert ist, ne. Dann habe ich eine Stabilität und kann so reindrücken. Und auf der anderen Seite habe ich dauerhaft das Gefühl, weil rechts meine starke Seite ist, drehe ich mich dann da nicht so rein, sondern immer noch so. Und dann habe ich immer das Gefühl, das ist definitiv so, dass ich nicht ganz so viel Druck ausüben kann. Da kann ich nichts gegen korrigieren. Das ist dann so." (IP_AB,ZZ.249-254).
} 
Zusätzlich zur antagonistisch-einseitigen Einleibung zwischen den Athleten und ihrem Sportgerät ist das Hineinspringen in den Schlitten durch eine Aneinanderreihung antagonistisch-einseitiger Einleibungen unter den Bobfahrern gekennzeichnet. Die zentrale Brücke dieser leiblichen Kommunikation sind hierbei die gegenseitigen Bewegungssuggestionen der Fahrer. Der Pilot ist derjenige, der das „vorgibt, den Einstieg, und das dann auf mich [= Position 2] überträgt, dann der Dreier-Mann und dann zum Schluss der Vierer“ (IP_BC, Z. 191f.). Was der Zweier-Mann hier mit dem Wort „überträgt“ beschreibt, ist die von dem in den Bob springenden Piloten ausgelöste und vom Zweier-Mann wahrgenommene Bewegungssuggestion, die sie beide ad hoc zu einer transleiblichen Einheit verbindet. Die sichtbare Bewegung des Piloten greift hier so dominant in den vitalen Antrieb des Zweier-Manns ein, dass dieser quasi ohne Reaktionszeit koagiert. Und diese antagonistisch-einseitige Einleibung (einseitig, weil der Pilot nicht auf den Zweier-Mann reagiert) setzt sich idealerweise fort: Der Zweier-Mann übt mittels seiner Bewegungen einen leiblichen Sog auf den Dreier-Mann aus, der von diesem Sog mitgezogen wird und selbst abspringt, was wiederum auf den Vierer-Mann eine bewegungssuggestive Kraft ausübt, sodass auch er in den Schlitten springt. Die leibliche Kommunikation der vier Athleten beim Hineinspringen erweist sich somit als eine Kette antagonistisch-einseitiger Einleibungen, die sich mittels bilateraler Bewegungsimpulse vollziehen. Ergänzend dazu ein Zitat eines Fahrers auf der Position 3:

Und dann irgendwann bewegt sich ja der Pilot vor mir direkt. Das sehe ich ja. [...] Und dann schiele ich so ein bisschen nach rechts und gucke dann, [...] wann der andere drauf geht und wenn ich merke an seinen Bewegungen, ne. Der [ZweierMann], mit dem bin ich schon oft gefahren. Da weiß ich so, wie das - da gucke ich da so drauf und dann sehe ich, was der macht. Und dann, ja. Ich kann dir nicht genau sagen, worauf $i c h$ da reagiere, wei $\beta$ ich nicht. Aber es ist wahrscheinlich so, man versucht immer als der linke Mann, wenn der rechte auf den Abweiser geht, ne, auf dieses Stück neben dem Schlitten, dann gehe ich auch drauf so. (IP_AB, Z. 206-216)

Im Fall dieses Teams befinden sich Position 1 und 3 auf derselben Seite des Bobs. Der Dreier-Mann sieht zwar die Bewegungen von der Eins so wie in dem vorherigen Zitat der Zweier-Mann, doch sind das in diesem Fall keine suggestiven Bewegungen, die den Dreier-Mann leiblich so in Beschlag nähmen, dass dieser sein Verhalten daran orientierte. Stattdessen „schielt“ der Dreier-Mann über den Schlitten hinweg zum Zweier-Mann und „merkt an seinen Bewegungen“, wann er selbst auf den Abweiser zu springen hat. Interessant ist hierbei, dass der DreierMann zwar sieht, was der Zweier-Mann macht, aber dennoch nicht weiß, ,worauf ich da reagiere“. Anscheinend ist es nicht so sehr die visuelle Wahrnehmung der Bewegung seines Partners, die ihn in antagonistisch-einseitiger Einleibung dazu drängt, die situativ erforderliche Bewegung auszuführen, sondern ein ganzheitlicher leiblicher Eindruck, den er spürbar wahrnimmt („merkt“) und mittels einer Eigenbewegung aufnimmt und fortführt. Dieses Koagieren ohne Reaktionszeit in solidarischer Einleibung ist hierbei entscheidend dadurch bedingt, dass die beiden Athleten offenkundig ein gut eingespieltes Team sind. ${ }^{13}$

\section{Hinsetzen}

Nach dem Hineinspringen in den Schlitten folgt als letzte Startphase das Hinsetzen. Wie in der vorangegangenen dritten Startphase ist auch hier die Abstimmung zwischen den Positionen 2 und 3 die wichtigste. Während der Pilot sich sofort hinsetzen kann, ohne auf seine drei Mitfahrer Rücksicht nehmen zu müssen, und der Bremser „eigentlich immer Platz" hat (IP_BC, Z. 281f.), müssen die Zwei und

\footnotetext{
13 Auch sehrgut eingespielte Teams sind jedoch nicht vor Zwischenfällen gefeit. So kann es passieren, dass auch ein erfahrener Pilot beim Einsteigen abrutscht (IP_BC, Z. 156-164, IP_BB Z. 178-181) oder er zwei Schritte zu kurz läuft (IP_AD, Z. 194f.), die Drei vor der Zwei in den Schlitten springt (IP_AC, Z. 143f.) oder der Bremser zu seitlich reinspringt, wodurch der Bob leicht verrutscht (IP_BD,Z.202-206).
}

Drei ihr Hinsetzverhalten leiblich und körperlich koordinieren. Dazu die Äußerung eines Fahrers auf der Zwei:

Und ich dann aber praktisch warte, bis [...] der Dreier-Mann, mit seinen Beinen bei mir vorbei ist und er gibt mir dann das Kommando „ab“. Das bedeutet, er ist in seiner Position. Jetzt kann nichts mehr passieren. Ich kann mich hinsetzen, weil sonst könnte es schon passieren, wenn ich mich zu zeitig hinsetze, dass er mit seinen Füßen, seinen Beinen, nicht mehr an der Seite vorbeikommt. Sprich, er würde dann im Schlitten drin hocken und das würde bedeuten, ich muss so schnell wie möglich wieder aufstehen, was gleichzeitig wieder bedeutet, dass wenn ich aufstehe, habe ich einen Kontakt zu [Position 1]. (IP_BC, Z. 262-232)

Der Zweier-Mann ist kurz vor dem Dreier-Mann in den Schlitten gesprungen, nutzt diesen zeitlichen Vorsprung jedoch nicht, um sich vor diesem hinzusetzen. Im Gegenteil, er verharrt für einen Moment in "gehockter Position, damit ich nicht so sehr im Wind stehe" (ebd., Z. 136f.) und „wartet“, bis der Dreier-Mann seine Beine an ihm vorbeigeschoben hat und ihm ein Kommando zum Hinsetzen gibt. (In anderen Teams gibt der Vierer-Mann das Hinsetzkommando. ${ }^{14}$ Der Vierer-Mann schließt sich diesem Kommando an, sodass sich idealerweise „alle drei Anschieber [..] gleichzeitig in den Schlitten setzen, um zu vermeiden, dass das praktisch wie eine Kettenreaktion ist und dass drei verschiedene Impulse in den Schlitten wirken“ (ebd., Z. 290-292).

Dieser Beschreibung zufolge vollzieht sich das Hinsetzen der Positionen 2, 3 und 4 als eine Aneinanderreihung von bilateralen leiblichen Kommunikationen, konkret als eine Folge antagonistisch-einseitiger Einleibungen, deren zentrales $\mathrm{Me}$ dium die von den Athleten bewirkten und wahrgenommenen Bewegungssug-

\footnotetext{
14 Das leibliche Kriterium für das Hinsetzkommando von einem Vierer-Mann ist dasselbe wie beim Dreier-Mann: „Sobald ich erahne, dass ich mit meinen Füßen schon an der Drei vorbei bin, rufe ich , ab' und dann geht das eigentlich relativ schnell. Bügel noch rein und dann sitzt du drin." (IP_BA, Z. 104f.; Herv. von Verf.) „Ahnen, spüren, wittern" sind "mantische" Formen leiblicher Kommunikation (Uzarewicz, 2011, S. 209ff.).
} 
gestionen sind: Die kurzfristig statische Körperposition des Zweier-Manns greift als leiblich dominanter Impuls in den vitalen Antrieb des Dreier-Manns ein, sodass dieser mit einer körperlichen $\mathrm{Be}$ wegung und einem kurzen Ausruf („ab“) darauf reagiert. Für das Gelingen dieser leiblich-körperlichen Abstimmung ist es entscheidend, dass die Beine des DreierManns am Zweier-Mann vorbeigeschoben sind, bevor dieser sich hinsetzt. Die Wahrnehmung der vorbeigeschobenen Beine ist hierbei vor allem eine leibliche und weniger eine visuelle: „Aber ich spüre das, wenn der Hintermann praktisch an mir vorbei geht" (IP_AC, Z. 118), ist eine wiederholt getätigte Äußerung in den Interviews. Die spürbare Wahrnehmung der Beine des Hintermanns ist auch bedeutsamer als der hörbare Laut „ab“, was allein daran erkennbar ist, dass er immer wieder überhört wird, der Zweier-Mann (und der Dreier-Mann, sofern das Kommando vom Vierer-Mann kommt) sich aber gleichwohl hinsetzt. Die sichtbare und hier für den Zweier-Mann ebenso spürbare Bewegung sowie der hörbare Laut des Dreier-Manns sind dann wiederum leiblich so machtvoll, dass sie den Zweier- und den Vierer-Mann im wörtlichen Sinn dazu bewegen, sich hinzusetzen. Die zentrale Aufgabe der drei Anschieber ist es dabei, sich gleichzeitig hinzusetzen, also ohne Reaktionszeit zu koagieren. Der Effekt dieser solidarischen Einleibung ist wiederum ein zeitlich relevanter, physischer Impuls:

Insgesamt wollen wir uns gleich hinsetzen, um einfach den Schlitten noch mal, wenn wir uns alle drei Mann mit über 300 Kilo noch mal in diesen Schlitten reinsetzen gleichzeitig, kriegt der noch mal beim Abgang so einen kleinen Push und das kann auch der Unterschied sein, dass die Abgangsgeschwindigkeit ein Stück höher ist bei uns. (IP_BB, Z. 200-204)

Bobteams, deren leiblich-kommunikative Abstimmung beim Hinsetzen misslingt, sodass nicht ,jeder so sitzt, wie er muss“, haben am Start „vielleicht schon die Geschwindigkeit dort ein bisschen verloren“ (IP_BD, Z. 151f.) und damit einen zeitlichen Nachteil, der im Rennen oft nicht mehr zu korrigieren ist. Beim Hinsetzen gilt es daher, mit einer gewissen leiblichen Spannung körperlich kontrolliert zu agieren und sich zum Beispiel nicht „einfach reinplumpsen [zu] lassen“, weil das im ungünstigsten Fall bedeutet, „dass der Bob, wenn man schon entsprechend weit ist, rausrutscht" aus der Spur (ebd., Z. 198-200). Auch damit ginge ein kaum mehr aufzuholender Zeitverlust bereits beim Start einher.

\section{Schluss}

Der vorliegende Beitrag ging von der Annahme aus, dass die Startphase beim Viererbob von entscheidender Bedeutung ist für eine gute Endzeit, weshalb es eine wichtige Frage ist, wie der Viererbobstart gelingt. Ziel war es daher zu verstehen, wie der Viererbobstart erfolgreich vollzogen wird. ${ }^{15}$ Die zentrale These lautete, dass für das Gelingen des Viererbobstarts die leibliche Kommunikation der Athleten untereinander wie auch mit ihrem Sportgerät, dem Bob, entscheidend ist. Die leibphänomenologische Interpretation der Interviews von acht Kaderathleten des BSD ergab, dass die Startphase aus vier aufeinander folgenden Teilphasen besteht - Einstimmen, Anschieben, Hineinspringen, Hinsetzen -, in denen sich leibliche Kommunikationsprozesse in zwei Varianten vollziehen, nämlich als antagonistische - mit den beiden Dimensionen einseitig und wechselseitig und solidarische Einleibung. Als zentrales empirisches Ergebnis lässt sich zweierlei festhalten:

(1) Für das Gelingen des Viererbobstarts ist die antagonistischeinseitige Einleibung der Athleten untereinander und mit dem Bob augenscheinlich wichtiger als die antagonistisch-wechselseitige

15 Obgleich das zentrale Ziel dieses Textes ein hermeneutisches war, lässt sich aus den Ergebnissen auch eine zentrale sportpraktische Schlussfolgerung ziehen. Unsere These jedenfalls lautet: Die Athleten und Trainer im Bobsport sollten nicht so sehr-wie wirin diesem Beitrag - auf die eigen- und transleiblichen Gelingensaspekte achten, sondern vor allem auf die subjektiv wahrnehmbaren Misslingensmomente. Das heißt, die leiblichen Irritationen, Dissonanzen, Differenzerfahrungen etc. der Fahrer sind erkenntnisrelevante Informationen, aus denen sich praxisrelevante Konsequenzen ziehen lassen.
Einleibung. Die Bobfahrer koordinieren ihre Bewegungen, indem sie insbesondere die Bewegungssuggestionen ihres Vorder-, Nebenoder Hintermannes wie auch die Bewegungsaufforderungen des Schlittens (vor allem des Abweisers und der Bordwand) leiblich wahrnehmen und unmittelbar körperlich beantworten. Die leibliche Kommunikation beim Viererbobstart ist somit eher ein monologischer (einseitiger) denn ein dialogischer (wechselseitiger) präreflexiver Abstimmungsprozess, der sich primär bilateral und nicht multilateral vollzieht. Für eine gelingende Gesamtkoordination der Startphase ist dabei entscheidend, dass sich die phasenspezifischen antagonistisch-einseitigen Einleibungen wie Glieder einer Kette nahtlos aneinanderreihen. Als größte Herausforderung - besonders für die Athleten auf den Positionen 2 und 3 - erweist sich hierbei die dritte Startphase, das Hineinspringen, weil hier der Schlitten am deutlichsten als mächtiger Antagonist (er appelliert und nötigt leiblich) erfahrbar wird.

(2) Die solidarische Einleibung ist sowohl Voraussetzung als auch Resultat eines gelingenden bzw. gelungenen Starts: Einerseits glückt der Bobstart umso eher, je besser das Team aufeinander eingestimmt ist, andererseits basiert die reibungsfreie Bewegungskoordination auf einer Reihe ein- und wechselseitiger antagonistischer Einleibungen. Der Beitrag bestätigt damit eine These von Schmitz: „Solidarische und antagonistische Einleibung mischen sich auch, z. B. in sportlichen Wettkämpfen von Mannschaften. Gemeinsam ist beiden Typen das Zusammenwirken ohne merkliche Reaktionszeit als Leitsymptom der Einleibung [...] “ (Schmitz, 2003, S. 40). Das zentrale Medium für die solidarische Einleibung im Sinne eines Koagierens ohne bewusst wahrnehmbare Reaktionszeit ist beim Viererbobstart der gemeinsame Bewegungsrhythmus, das 
zentrale Ziel solidarischer Einleibung in allen Phasen ist das richtige Timing.

Vor dem Hintergrund, dass die Sportwissenschaft in ihren wenigen Untersuchungen zum Bobstart bislang ausschließlich die Körperlichkeit der Athleten und den technischen Gegenstand dieses Sports, den Bob, thematisiert hat, hat dieser Beitrag mit seinem Fokus auf die Leiblichkeit und leibliche Kommunikation der Athleten einen vollkommen neuen, u.E. erkenntnisreichen Aspekt in die sportwissenschaftliche Diskussion zum Bobsport eingebracht. Ebenso gewinnbringend erscheint uns der in der Sportwissenschaft bis dato nahezu unbekannte neophänomenologische Ansatz von Schmitz, dessen differenziertes Begriffsinstrumentarium die hier vorgelegte Mikroanalyse des Viererbobstarts zuallererst ermöglichte. Der Text hat gezeigt, dass eine große Stärke des neophänomenologischen, Werkzeugkastens' darin besteht, leiblich-kommunikative Prozesse zwischen Athleten sowie zwischen ihnen und den Dingen (Bordwand, Abweiser) und Halbdingen (Musik) sportlicher Praxis detailliert rekonstruieren $\mathrm{zu}$ können. Der größte Erkenntnisgewinn dieses Textes liegt hierbei womöglich darin, nachgewiesen zu haben, dass sich und auf welche Weise sich leibliche Kommunikation beim Viererbobstart nicht nur zwischenmenschlich, sondern ebenso transhuman vollzieht.

Mit seinem theoretisch-konzeptionellen Fokus auf leibliche Kommunikation im Sport liefert der Beitrag über den Bobsport hinaus einen innovativen Beitrag zur phänomenologischen Sportforschung. Phänomenologische Abhandlungen zum Sport fokussieren nämlich primär - Ausnahmen (z. B. Meyer \& Wedelstaedt, 2017) bestätigen die Regel - die subjektiven Erfahrungen und sinnlichen Wahrnehmungen im Sport. Dies hat zur Konsequenz, dass „[...] at present, there is a relativ lacuna in terms of research that synthesis the phenomenological and the interactional" (AllenCollinson \& Evans, 2019, S. 298). Indem dieser Text dargelegt hat, wie eine solche phänomenologisch-interaktionistische Synthese aussehen kann, hat er grund- sätzlich verdeutlicht, dass Philosophie und empirische (Sport-)Wissenschaft sich keineswegs ausschließen, sondern im Gegenteil sich fruchtbar ergänzen können. Daher versteht sich dieser Beitrag auch als ein Appell an die Sportwissenschaft, öfter und konsequenter den Schulterschluss von Philosophie und empirischer Forschung im Sinne einer empirischen Sportphilosophie - oder allgemeiner: einer ,theoretischen Empirie“ (Kalthoff, Lindemann, \& Hirschauer, 2008) - zu suchen.

\section{Korrespondenzadresse

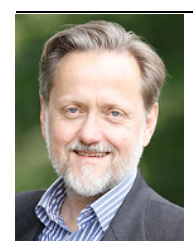 \\ Prof. Dr. Robert Gugutzer \\ Institut für Sportwissen- schaften, Goethe-Universität Frankfurt a.M. \\ Ginnheimer Landstr. 39, 60487 Frankfurt a.M., Deutschland gugutzer@sport.uni- frankfurt.de}

Danksagung. Wir danken den Gutachter*innen für ihre konstruktive Kritik am Originalmanuskript.

Funding. Open Access funding enabled and organized by Projekt DEAL.

\section{Einhaltung ethischer Richtlinien}

Interessenkonflikt. A. Elm und R. Gugutzer geben an, dass kein Interessenkonflikt besteht.

Für diesen Beitrag wurden von den Autoren keine Studien an Menschen oder Tieren durchgeführt. Für die aufgeführten Studien gelten die jeweils dort angegebenen ethischen Richtlinien.

Open Access. Dieser Artikel wird unter der Creative Commons Namensnennung 4.0 International Lizenz veröffentlicht, welche die Nutzung, Vervielfältigung, Bearbeitung, Verbreitung und Wiedergabe in jeglichem Medium und Format erlaubt, sofern Sie den/die ursprünglichen Autor(en) und die Quelle ordnungsgemäßnennen, einen Link zur Creative Commons Lizenz beifügen und angeben, ob Änderungen vorgenommen wurden.

Die in diesem Artikel enthaltenen Bilder und sonstiges Drittmaterial unterliegen ebenfalls der genannten Creative Commons Lizenz, sofern sich aus der Abbildungslegende nichts anderes ergibt. Sofern das betreffende Material nicht unter der genannten Creative Commons Lizenz steht und die betreffende Handlung nicht nach gesetzlichen Vorschriften erlaubt ist, ist für die oben aufgeführten Weiterverwendungen des Materials die Einwilligung des jeweiligen Rechteinhabers einzuholen.
Weitere Details zur Lizenz entnehmen Sie bitte der Lizenzinformation auf http://creativecommons.org/ licenses/by/4.0/deed.de.

\section{Literatur}

Allen-Collinson, J., \& Evans, A.B. (2019). To be or not to be phenomenology: that is the question. European Journal for Sport and Society, 16,295-300.

Alloa, E., Bedorf, T., Grüny, C., \& Klass, T.N. (Hrsg.). (2012). Leiblichkeit. Geschichte und Aktualität eines Konzepts. Tübingen: Mohr Siebeck.

Bedorf, T. (2017). Selbstdifferenz in Praktiken. Phänomenologie, Anthropologie und die korporale Differenz. In T. Bedorf \& S. Gerlek (Hrsg.), Phänomenologie und Praxistheorie (S. 57-75). Hamburg:Meiner.

Boerboom, S., Hainzlmaier, C., Kraus, F., \& Wintermantel, E. (2006). Tribologie im Stahl-Eis-Kontakt: Einfluss des Druckes. In K. Witte, J. EdelmannNusser, A. Sabo \& E. F. Moritz (Hrsg.), Sporttechnologie zwischen Theorie und Praxis IV (S.65-71) Aachen: Shaker.

Böhme, G. (2003). Leibsein als Aufgabe. Leibphilosophie in pragmatischer Hinsicht. Kusterdingen: Die GraueEdition.

BSDDerSport.https://www.bsd-portal.de/sport/bob/ der-sport/.Zugegriffen:31. Aug. 2020.

Cross, M. R., Tinwala, F., Lenetsky, S., Samozino, P., Brughelli, M., \& Morin, J.-B. (2017). Determining friction and effective loading for sled sprinting. Journal of Sport Sciences, 35, 2198-2203.

Dabnichki, P.(2015). Bobsleigh performancecharacteristics for winning design. Procedia Engineering, $112,436-442$.

Dabnichki, P. (2016). Computer analysis of bobsleigh team push. In P. Chung, A. Soltoggio, C. W. Dawson, Q. Meng \& M. Pain (Hrsg.), Proceedings of the 10th International Symposium on Computer Science in Sport (ISCSS) (S. 193-200). Cham: Springer.

Elm, A. (2020). Mensch-Maschine-Interaktion im Bobsport. Eine leibphänomenologische Untersuchung. Unveröffentlichte Masterarbeit am Institut für Sportwissenschaften der GoetheUniversität Frankfurt am Main.

Ewald, U. (2015). Vertikale Erlebnisse. Ein erweitertes Raumverständnis, aufgezeigt am Beispiel des Hallenkletterns. In M. Großheim, A. K. Hild, C. Lagemann \& N. Trcka (Hrsg.), Leib, Ort, Gefühl. Perspektivem der räumlichen Erfahrung (S.345-368). Freiburg, München:Alber.

Freimüller, P. A., Biener, K., \& König, M. F. (2000). Verletzungen und Risiken im Bobsport. Schweizerische Zeitschrift für Sportmedizin und Sporttraumatologie, 48, 154-157.

Fuchs, T. (2000). Leib, Person, Raum. Entwurf einer phänomenologischen Anthropologie. Stuttgart: Klett-Cotta.

Gerber, A. (1987). Das Training des Bobfahrers. Hornbrechtikon: Selbstverlag

Goldmann, J.-P., Braunstein, B., Sanno, M., Städule, B., Göll, F., Grothe, S., Droszez, A., \& Albracht, K. (2018). Komplexe biomechanische Analyse des Bobanschubs unter Berücksichtigung der tendinösen und neuromuskulären Eigenschaften der unteren Extremität. In BISp-Jahrbuch Forschungsförderung 2017/2018 (S. 339-347). Bonn: Bundesinstitut für Sportwissenschaft.

Gugutzer, R. (2015a). (Neo-)Phänomenologie der Sportsucht. Theoretische und empirische 
Annäherungen. Sport und Gesellschaft - Sport and Society, 12, 189-214.

Gugutzer, R. (2015b). Leibliche Interaktion mit Dingen, Sachen und Halbdingen. Zur Entgrenzung des Sozialen (nicht nur) im Sport. In H. K. Göbel \& S. Prinz (Hrsg.), Die Sinnlichkeit des Sozialen. Wahrnehmung und materielle Kultur (S. 105-122). Bielefeld: transcript.

Gugutzer, R. (2015c). Public Viewing. Ein sportiv gerahmtes kollektivleibliches Situationsritual. In R. Gugutzer \&M. Staack (Hrsg.), Körper und Ritual. Sozial- und kulturwissenschaftliche Zugänge und Analysen (S.71-96). Wiesbaden: SpringerVS.

Gugutzer, R. (2016). Self-tracking als ZeitgeistPhänomen. In S. Duttweiler, R. Gugutzer, J.H. Passoth \& J. Strübing (Hrsg.), Leben nach Zahlen. Self-tracking als Optimierungsprojekt? (S. 161-182). Bielefeld: transcript.

Gugutzer, R. (2019). Moden des Sports - Manifestationen des Zeitgeists. In H. Busche \& Y. Förster (Hrsg.), Mode als ein Prinzip der Moderne? Ein interdisziplinärer Erkundungsgang (S. 81-102) Tübingen:Mohr.

Gugutzer, R. (2020). Atmosphären, Situationen und der Sport. Ein neophänomenologischer Beitrag zur soziologischen Atmosphärenforschung. Zeitschrift für Soziologie, 49, 371-390.

HainzImeier, C., Wolf, S., \& Wintermantel, E. (2005). FEM-Modellierung des Kufe-Eis-Kontaktes im Bobsport. In H. Gabler, U. Göhner \& F. Schiebl (Hrsg.), Zur Vernetzung von Forschung und Lehre in Biomechanik, Sportmotorik und Trainingswissenschaft (S. 204-208). Hamburg: Czwalina.

Handschin, T. Allgemeine Informationen Bobsport http://www.thomas-handschin.ch/bobsport/ allgemeines/index.php. Zugegriffen: 20. Sept. 2019.

Hartnack, F. (2017). Doing Gender und Feeling Gender im Sportunterricht: Eine leibphänomenologische Ethnografie des spielerischen Zweikämpfens. Göttingen:V\&RUnipress.

Heidegger, M. (2006).Sein und Zeit(19. Aufl.). Tübingen: Niemeyer.

IBSF (2020a). Bobsleigh history. https://www.ibsf.org/ en/our-sports/bobsleigh-history. Zugegriffen: 2.Jan. 2021.

IBSF (2020b). International Bobsleigh rules 2020. https://www.ibsf.org/images/federation/ Rules_and_Regulations/2020_International Rules_BOBSLEIGH_changes_highligted_26_ Oct_20.pdf.Zugegriffen:2.Jan. 2021.

IBSF (2020c). International women's monobob rules 2020. https://www.ibsf.org/images/federation/ Rules_and_Regulations/IBSF_International_ Womens_Monobob_Rules_2020_E.pdf. Zugegriffen:2.Jan. 2021.

Kalthoff, H., Lindemann, G., \& Hirschauer, S. (Hrsg.). (2008). Theoretische Empirie. Zur Relevanz qualitativer Forschung. Frankfurt a.M.:Suhrkamp.

Kümmel, J., Bergmann, J., Prieske, O., Krüger, T., Granacher, U., \& Gruber, M. (2015). Verbesserung der reaktiven Bewegungsleistung durch Postactivation Potentiation (PAP) und Motor Imagery (MI) bei Spitzenathletinnen und -athleten im Bobsport. In BISp Jahrbuch Forschungsförderung 2013/2014 (S. 131-135). Bonn: Bundesinstitut für Sportwissenschaft.

Leopold, G. (2020). Ansätze zur Erneuerung des Krafttrainings im Bobsport. In F. Lehmann, U. Wenzel \& I. Sandau (Hrsg.), Kräftiger, schneller, ausdauernder - Entwicklung der muskulären Leistung im Hochleistungssport (S. 99-105). Aachen:Meyer \& Meyer.
Meyer, G. (2008). Die Wertigkeit der koordinativen und konditionellen Bestandteile innerhalb der komplexen Schnelligkeitsleistung, untersucht an einer Pilotstudie zur zyklischen Beschleunigungsphase im Bobsport. In D. Lühnenschloß \& P. Wastl (Hrsg.), Quo vadis olympische Leichtathletik? Probleme, Bilanzen, Perspektiven (S. 157-166). Hamburg: Czwalina.

Meyer, C., \& von Wedelstaedt, U. (Hrsg.). (2017). Moving bodies in interaction -interacting bodies in motion.Intercorporeality, interkinesthesia, and enaction in sports. Amsterdam: Benjamins.

Park, S.-H., Lim, S.-T., \& Kim, T.-W. (2019). Measurement of electromyography during bobsleigh pushstart: a comparison with world top-ranked athletes. Science \& Sports, 34, e25-e30.

Pernpeintner, A., \& Winkler, A. (2010). Lessons learned from the aerodynamic shape development process of a bobsleigh. Procedia Engineering, 2, 2407-2012.

Poirier, L., Lozowski, E. P., Maw, S., Stafnyshyn, D. J., \& Thompson, R.I. (2011). Experimental analysis of ice friction in the sport of bobsleigh. Sports Engineering, 14,67-72.

Rappe, G. (2018). Einführung in die moderne Phänomenologie. Phänomen - Leib - Subjekt. Bochum Freiburg: projekt verlag.

Schmitz, H. (1965). Teil 1:Der Leib. Bd. 2. Bonn:Bouvier

Schmitz, H. (1985). Phänomenologie der Leiblichkeit In H. Petzold (Hrsg.), Leiblichkeit. Philosophische, gesellschaftliche und therapeutische Perspektiven (S.71-106). Paderborn: Junfermann.

Schmitz, H. (1990). Der unerschöpfliche Gegenstand. Grundzüge der Philosophie. Bonn: Bouvier.

Schmitz, H. (2003). Was ist Neue Phänomenologie? Rostock: Koch.

Schmitz, H. (2009). Kurze Einführung in die Neue Phänomenologie. Freiburg, München: Alber.

Schmitz, H. (2011).Der Leib. Berlin, Boston: De Gruyter

Schmitz, H. (2019). New Phenomenology. A brief introduction. Milan: Mimesis International. Transl. by Rudolf $\mathrm{O}$. Müllan with support from Martin Bastert. With an introduction by Tonino Griffero

Schwere Jungs. (Deutschland 2006). Regie: M. Rosenmüller. DVD Constantin Film 2006

Staack, M. (2019). Figthing as real as it gets. A microsociological encounter. Berlin: J.B. Metzler.

Uzarewicz, M. (2011). Der Leib und die Grenzen der Gesellschaft. Eine neophänomenologische Soziologie des Transhumanen. Stuttgart: Lucius \& Lucius.

Winkler, A., \& Pernpaintner, A. (2010). Automated aerodynamic optimization of the position and posture of a bobsleigh crew. Procedia Engineering, 2,2399-2405.

Winkler, A., \& Pernpeintner, A. (2008). Improving the performance of a bobsleigh by aerodynamic optimization. In M. Estivalet \& P. Brisson (Hrsg.), The engineering of sport (Bd. 2, S. 329-338). Paris: Springer.

Witzel, A., \& Reiter, H. (2012). The problem-centered interview. Los Angeles: SAGE. 